\title{
Exploring the virtual space of Academia
}

\author{
Maria Menendez; Antonella de Angeli; Zeno Menestrina \\ Department of Information Engineering and Computer Science (DISI) \\ University of Trento Via Sommarive, 5 I-38123 POVO - Trento (Italy) \\ \{menendez; deangeli\}@disi.unitn.it; zeno.menestrina@studenti.unitn.it
}

\begin{abstract}
The aim of this chapter is to provide a view on how researchers present themselves in a social network specifically developed for supporting academic practices, how they share information and engage in dialogues with colleagues worldwide. We analysed data from 30,428 who have registered on a publicly available website to study the effect of academic position, university ranking and country on people's behaviour. Results suggest that the virtual network closely mirrors physical reality, reproducing the same hierarchical structure imposed by position, ranking, and country on user behaviour. Despite the potential for bridging and bonding social capital the networks has not achieved substantial changes in structures and practices of the academic context. Furthermore, our analysis highlights the need of finding new strategies to motivate the users to contribute to the community and support equal participation, as so far the community is mainly exploited as a static website.
\end{abstract}

\section{Introduction}

A consistent corpus of research in social networks has linked this technology to social capital, the value derived from being member of a community $[2,7,17]$. Social networks can increase the establishment and maintenance of weak-ties by bridging social capital and have some positive effect also on strong ties by bonding social capital [17]. Social capital is an important personal and organisational asset. It tends to improve processes of knowledge management and information sharing [7], a desirable outcome in many professional contexts, and in particular in research work. In this chapter, we provide some insights on how researchers have structured themselves into an on-line community specifically developed to support academic practices. In particular, we look at self-presentation strategies, information sharing, and on on-going dialogues supported by the community. This knowledge can facilitate our understanding of how technology shapes organiza- 
tional practices and how to design technology for supporting positive changes in existing structures [14].

The chapter is structured as follows. First, we review related work on the use of social networks in professional contexts with an emphasis on barriers and drivers in the adoption. Then, we present the main social networking websites for academics available on the web and present methods and results of our study. Finally, we discuss the findings and propose directions for future work.

\section{From personal to professional networks}

In the last decade, many different types of social networks have appeared on the Internet with some astonishing success stories. Facebook is the winner in terms of users and number of academic studies $[4,6,8,10,16,18]$. As for 2011, it entered the life of 800 millions people worldwide. Facebook has initially established as a tool for university students, but nowadays it pervades many other population segments. The move into the workplace has not been easy due to several tensions between personal and professional life. For instance, social networks challenge the organisational requirement to maintain confidentiality within the firewall, and the individual requirement to keep personal and professional life separated [16]. Enterprises usually have strict policies on data protection and security. A recent study commissioned by the Internet Security Company Webroot revealed that over $50 \%$ of American and British business with up to 500 employees has restricted access to any public social network. Yet, social networks may play an important and productive role in organisations. They can contribute to building social capital [17] and facilitate knowledge management and information sharing processes [7].

To lessen security threats and the difficult overlapping between professional and personal life, a number of ad-hoc social networks have been developed to be used within the company intranet $[3,5,15,17]$. Research, however, suggest that adoption and contribution rates are far from satisfactory. For instance Beehive, the internal IBM social network, collected registration from some $15 \%$ of the total employees over 2 years, and only some $30 \%$ of registered users visited the networks in a month [17]. Several studies have investigated motivational factors related to participation in social networking. Results highlight five basic dimensions addressing different needs related to functional, organizational, social, personal and technological factors.

Sharing and obtaining information are reported as main functional drivers for joining and contributing to professional social networks [20]. This source of motivation can be enhanced by individual predispositions and organisational culture which foster a perception of knowledge as a public good, belonging not to individuals, but to the whole organization [1]. Another important functional driver is the creation of new contacts, maintenance of old connections, and finding domain experts $[1,5,15]$. Surveillance of people's activity in the network has also been 
mentioned as a functional driver, especially at the managerial level of the organization [1]. However, some people perceive social networks as a waste of time [3].

Social factors facilitating adoption are linked to attraction by similarity. Users join groups and networks with whom they share some interests and passions [13, 17], and avoid places with content that they consider unsuitable [1, 19]. Personal exposure has been identified both as a driver and a hindrance. For some users, it increases visibility [1], opening opportunities for obtaining quality feedback on personal work [1] and self-expression [1, 19]. However, personal exposure can also be perceived as a privacy thread, preventing users from joining and contributing to social networks $[1,15]$. Technological factors also play an important role in adoption. Suitability of functionalities [19] drives users to join and use social networks, while accessibility issues, such as registration requirements, network security, and usability issues, $[1,16]$ are listed within the technology related barriers.

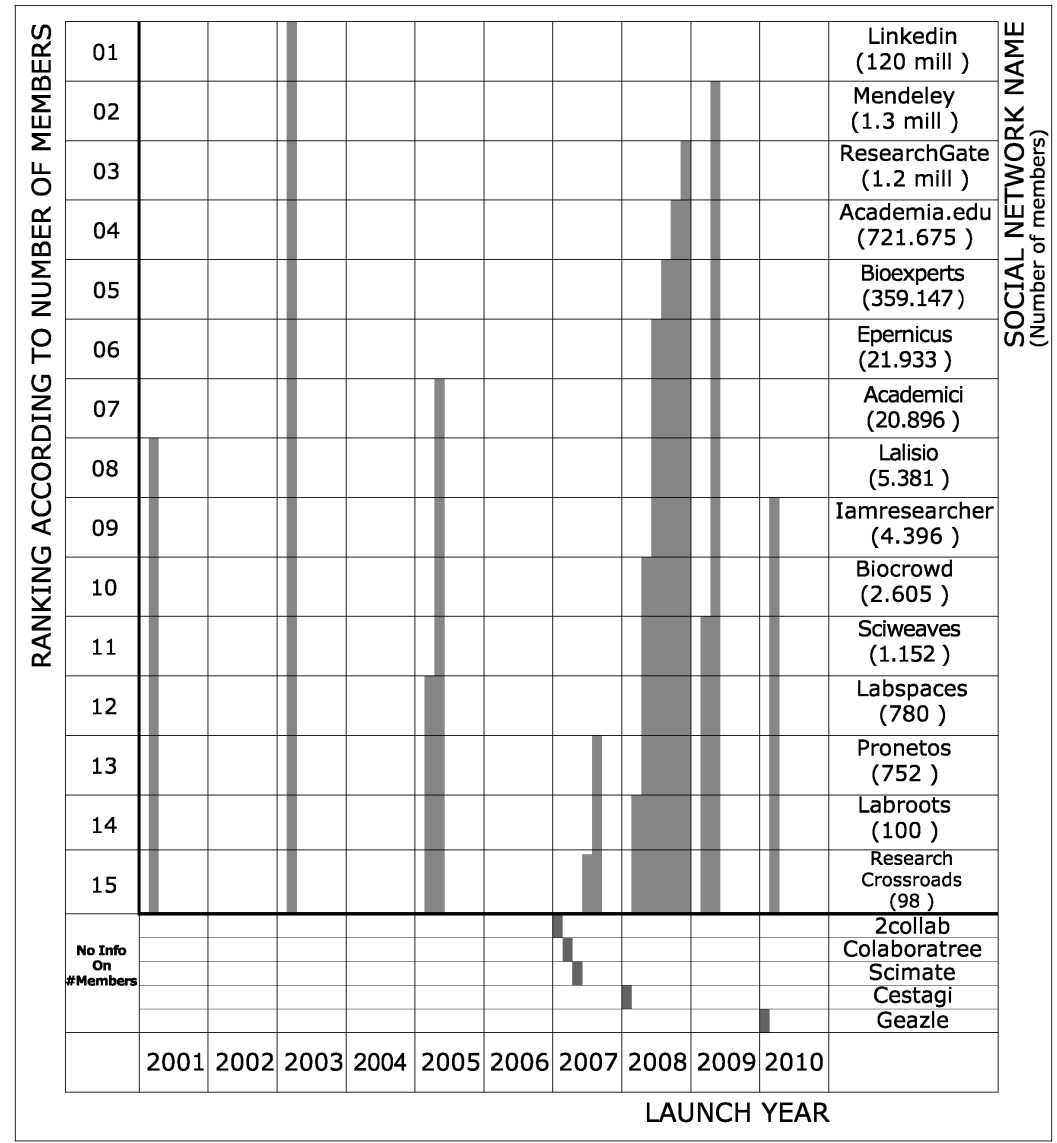

Fig. 1. List of Social Networks for researchers with ranking and launch year 


\section{Social networks for researchers}

In the last years there has been an increasing number of public social networks targeted to professionals, in particular to academics and researchers. Most of these sites aim at creating communities of professionals, where users can build their social networks by connecting to colleagues, finding old connections, and creating new links with professionals relevant to their work. Most of these social networks also aim at increasing personal visibility by creating online profiles and sharing work-related content $[12,16]$. Figure 1 illustrates the establishment of academics social network in time, and provides (where available) information on the number of users as for October 2011. Lalisio was one of the first public social network target to researchers, but it was not until Linkedin was launched that public social networks for professional became popular. Linkedin was not specifically targeted to researchers, but the community quickly adopted it. The period between 2007 and 2008 was especially active in the creation of social networks for researchers. Many of them (e.g., labspaces, pronetos, colaboratree, bioexperts, biocrowd, and labroots) focus on Life Sciences, such as Biology and Medicine. These fields of research were the ideal targets for social networks, as they require exchange of complex experimental protocols and expensive datasets. Furthermore, in these fields it is important that not only the significant findings, which are usually published in traditional academics journals, but also the failures reach the community in order to save funding and time.

Despite the growing number of social networks for researchers little is known yet on the specific characteristics of these communities. This chapter attempts to fill this gap by presenting a detailed analysis of use of Academia. In particular, we aim to answer the following research questions: Who are the users of social networks for academics and researchers? How do they use these social networks? Do individual, organizational, and cultural characteristics affect how they use them?

\section{Method}

As a case study for our investigation we selected Academia.edu as it as it allows tracking individual contributions to the community in a 'wall' available on the participants home page, following the typical Facebook metaphor. Academia.edu is advertised as a "platform for academics to share and follow research" and it was launched in October 2008. Every user has a personal webpage with information such as name, university, department or thesis title, and an open text field where they can write personal content. Name and surname are compulsory fields. University, department, and position are compulsory fields, unless the user registers as "independent researcher". 
The webpage also contains research interests: primary and secondary (at registration users have to state, at least, one primary interest). Users can upload documents such as books, papers, talks, teaching documents, blog posts, CV, and websites to their pages. Documents can be, although not necessarily, tagged with research interests. Recent updates (up to 25 actions) are automatically shown in every webpage. Examples of updates are: " $x$ followed the research interest: Web design", " $x$ answered "What do you use Academia .edu for?"”, and "X started following the work of 8 people"). Explicit connections among users are represented by department networks, followers, and followees. Department networks are automatically created by the system clustering all the users working at the same department, and can be manually adjusted by the users. Users can also connect to external people by following them, without needing permission from the followed person. Users can interact with other community members by posting questions and answers. For a question to be posted, it needs to be tagged with at least one research interest. Questions and other content related items, such as paper and books, can also be followed.

\section{Procedures}

Data were automatically collected between the $1^{\text {st }}$ and the $16^{\text {th }}$ of May 2011 using an algorithm developed within the group, and stored in off-line databases. Data were anonymized and no information which allowed retrieving users' identity was stored. Data collection was divided in three stages. During the first stage, user's webpages were manually analyzed to identify important information elements to be used in the analysis. In total, 33 variables were selected to describe information contained in webpages. These variables include information on user profile (e.g., position and affiliation), absence/presence of specific fields (e.g., picture, email address, and CV), number of elements per field (e.g., number of research interests, followers, and papers), and activity information (e.g., date of the first and last update). In the second stage, the identified variables were collected from users affiliated to a medium-size European University and to a large North American University. Data from 147 and 1295 users respectively were collected. This first data collection evaluated the potential of the variables to generate relevant user information. The variables showed interesting information on user's profile, activity, and content. However, the method used for data collection limited the potential of the dataset by bounding the affiliations to a predefined list.

During the last stage, a larger data set was collected. In order to overcome the limitations of the previous method, data were collected by research interest. Eight interests from different academic disciplines were selected to cover a broad range of fields (i.e., Anthropology, Biology, Biomedicine, Chemistry, Computer Science, Medical Sciences, Medicine, and Philosophy). Data from 30,428 users following, at least, one of the eight research interests were collected. 


\section{Variables}

The independent variables used in this study are information contained in, or computed from, compulsory registration fields. In particular we took into consideration the researcher position, the human development index of the country where the researcher was affiliated and the affiliation ranking according to the Times Higher Education. These factors were used to analyse variations on a set of indices created by combining conceptually related variables. All these variables were retrieved by facultative fields contained in the user's webpage, as we wanted to analyze variations in the quantity of information voluntarily provided. A summary description of independent and dependent variables is reported in Table 1. Independent variables are detailed in the remaining of this paragraph, whereas a description of each dependent variable is reported in the result section.

\begin{tabular}{|c|c|c|}
\hline Category & Factors & Collected/computed variables \\
\hline \multirow[t]{3}{*}{ Independent variables } & Position & User's Position \\
\hline & Ranking & $\begin{array}{l}\text { Absence/presence of affiliation } \\
\text { in the best } 400 \text { Universities }\end{array}$ \\
\hline & Country development & $\begin{array}{l}\text { Affiliation country clustered by } \\
\text { Human Development Index } \\
\text { (HDI) }\end{array}$ \\
\hline \multirow[t]{8}{*}{ Dependent variables } & Indices & Collected/computed Variables \\
\hline & Personal information & $\begin{array}{l}\text { Picture, Status, About, CV, } \\
\text { Homepage }\end{array}$ \\
\hline & Content contribution & $\begin{array}{l}\text { NPaper, NBooks, NTalks, NTeach- } \\
\text { ingDocuments, NBlogs, NWebsites }\end{array}$ \\
\hline & Research interests & $\begin{array}{l}\text { NPrimaryResearchInterests, } \\
\text { NSecondaryResearchInterests }\end{array}$ \\
\hline & Openness to contact & $\begin{array}{l}\text { Homepage, Address, Phone, Skype } \\
\text { ID, email }\end{array}$ \\
\hline & Popularity & NFollowedby - NFollows \\
\hline & Interaction among users & $\begin{array}{l}\text { NAskedQuestions, } \\
\text { NAnsweredQuestions, NFollowing } \\
\text { Questions }\end{array}$ \\
\hline & Level of engagement & Day's interval, NUpdates \\
\hline
\end{tabular}

Table 1. Variables categorized by dependent and independent.

\section{Position}

At sign up users could select their position from a list containing "faculty member", "post-doc", "graduate student", “emeritus/emerita", "undergraduate", 
"alumnus/alumna" or "independent researcher". Alternatively, they could enter a label in a blank field. Some $97 \%$ of the users selected their position from the list. For the sake of simplicity, the remaining 3\% was not included in the analysis. Furthermore, users whose position suggests not direct professional use of the system were not included in the analysis (i.e., "Emeritus/emerita", "undergraduate", and "alumnus/alumna"). This led to the deletion of some $15 \%$ of the users. The sample was finally composed of graduated students $(49 \%)$, faculty members $(36 \%)$, independent researchers $(9 \%)$, and post-docs $(6 \%)$.

\section{Country development}

As no information on the country of the affiliation was collected at sign up, we evinced this information from the content manually entered by the user in the field affiliation. These data were very noisy, due to the open question style. Thus, the text was standardized by a string search tool, which correctly identified $94 \%$ of the affiliations. The standardized affiliations were matched to a country by an external web service ${ }^{1}$, which automatically covered some $70 \%$ of the sample, with a very high reliability as evinced by a human rater performing double coding on some $10 \%$ of the sample. The remaining entries were checked manually, allowing disambiguation of some $94 \%$ of the standardized affiliations (99\% of the users). Most of the uncovered entries were international companies or Universities based in more than one country.

The dataset included 142 countries. The majority of the users worked for research institutions in the USA (31\%), UK (13\%), India (8\%), Canada (5\%), and Brazil (3\%). Overall North America accounted for some $38 \%$ of the people, Europe $21 \%$ and Asia $6 \%$. The remaining $9 \%$ was evenly spread between Central and South America, Oceania, and Africa. A country development index was computed by using the country's Human Development Index (HDI) ${ }^{2}$. A large part of the users were affiliated in very-high developed countries $(72 \%)$, followed by users in high developed countries (13\%), medium (13\%) and low developed countries $(2 \%)$. Given the very skewed distribution of the country development index, medium and low developed countries were clustered together, to be used as independent variables in the analysis.

\section{Ranking}

A University ranking index was calculated by matching the standardized affiliation name to the list of the best 400 Universities published by the Times Higher

\footnotetext{
${ }^{1}$ http://univ.cc

2 http://hdr.undp.org
} 
Education $^{3}$. Some $9 \%$ of the users' affiliations appeared in the list. Frequency and percentage of users affiliated to ranked Universities are reported in Table 2, grouped by 8 intervals. A dichotomous (presence vs. absence) variable was finally computed, to be used in the analysis.

\begin{tabular}{|c|c|c|}
\hline $\begin{array}{c}\text { Ranking in the list of the best } \\
\text { 400 Universities }\end{array}$ & Frequency & Percentage \\
\hline $1-50$ & 3877 & 35 \\
\hline $51-100$ & 1550 & 14 \\
\hline $101-150$ & 1464 & 13 \\
\hline $151-200$ & 1144 & 10 \\
\hline $201-250$ & 904 & 8 \\
\hline $251-300$ & 822 & 8 \\
\hline $301-350$ & 776 & 7 \\
\hline $351-400$ & 477 & 4 \\
\hline
\end{tabular}

Table 2. Academia.edu affiliations distributed by university ranking (excl. Affiliations with no ranking and independent researchers)

\section{Results}

The dependent variables were analysed by a set of one-way analysis of variance with Position (4), Country development (3) and Ranking (2) as between subjects variables. Due to the fact that the factors subsume different sample size (e.g., independent researchers did not report a country) and that ranking and country development are strongly correlated, a full factorial design was not possible. Posthoc comparisons were based on the Least-Significance Difference method. Partial eta-squared $\left(\eta_{\mathrm{p}}^{2}\right)$ was used as an estimate of effect size. As a general guideline, $\eta_{\mathrm{p}}{ }^{2}=.01$ are considered small, $\eta_{\mathrm{p}}{ }^{2}=.06$ medium, $\eta_{\mathrm{p}}{ }^{2}=.14$ large.

\section{Personal Information}

A personal information index was computed by adding a value of 1 for each piece of information provided by the user to the fields: picture, homepage, about, status, and $\mathrm{CV}$. The index ranged from 5 (all information provided) to 0 (no information provided). The mean was $1.02(\mathrm{SD}=1.19)$, but some $44 \%$ of the users did not provide any personal information. Overall, Pictures were the most frequently upload-

\footnotetext{
${ }^{3}$ www.timeshighereducation.co.uk
} 
ed items (47\%), followed by Homepage (18\%), About (16\%), Status (13\%), and CV $(9 \%)$.

The one-way analysis of variance returned a significant effect of position ( $F$ $\left.(3,30424)=162.25, p<.001, \eta_{\mathrm{p}}{ }^{2}=.02\right)$, country development $\left(F_{(2,26534)}=280.56, p<\right.$ $\left..001, \eta_{\mathrm{p}}{ }^{2}=.02\right)$, and ranking $\left(F_{(1,27570)}=107.01, p<.001, \eta_{\mathrm{p}}{ }^{2}=.04\right)$ on personal information. On the average, people affiliated to ranked universities disclosed more information than people affiliated with non-ranked universities. As regards the effect of position, post-hoc tests indicated a scale with significant intervals between faculty members, who submitted more information, post-doc, graduate students, and independent researchers. The effect of country development was due to users affiliated in very high developed countries which shared more information than users in high and medium-low developed countries, and users in high developed countries who shared more information than users affiliated in low-medium developed countries. Among the very high developed countries, we compared researchers in the US and Europe. A significant effect of location $\left(F_{(2,19032)}=47.61\right.$, $\left.p<.001, \eta_{\mathrm{p}}{ }^{2}=.005\right)$ emerged as Europeans disclosed more personal information than North Americans.

\section{Content contribution}

A content contribution index was computed by adding all items uploaded relatives Papers, Books, Talks, Teaching Documents, Blogs, and Websites. On the average, users uploaded some 4.55 files $(S D=10.05)$. Some $10 \%$ of the users did not upload any file. Users most often contributed papers (23\%), non-personal websites $(10 \%)$, books $(6 \%)$, talks $(4 \%)$, teaching documents $(2 \%)$, blogs $(1 \%)$. There was a positive correlation between personal information and content contribution indices $\mathrm{r}=.38 ; \mathrm{p}<.01$, highlighting a general predisposition to contribution in both variables.

The quantity of content contributed to the community varied as a function of position $\left(F_{(3,30424)}=435.01, p<.001, \eta_{\mathrm{p}}{ }^{2}=.04\right)$, country development $\left(F_{(2,26534)}=\right.$ $\left.128.28, p<.01, \eta_{\mathrm{p}}{ }^{2}=.01\right)$, and university ranking $\left(F_{(1,27570)}=55.4, p<.001, \eta_{\mathrm{p}}{ }^{2}=\right.$ $.002)$. The post-hoc test on position indicated that faculty members contributed significantly more than post-docs, who contributed more than graduate students and independent researchers. The post-hoc test on development indicated a scale with significant intervals between pairs, starting from user affiliated in very-high developed countries, who contributed more content, to users in high developed countries and medium-low developed countries. Among the very-high developed countries, there was an effect of location on the amount of contributed content $\left(F_{(2,19032)}=33.95, p<.001, \eta_{\mathrm{p}}^{2}=.004\right)$. Europeans contributed more than North Americans. People affiliated to ranked universities contributed more material than people affiliated to non-ranked universities. 


\section{Research Interest}

The research interest index was computed by adding all items indicated as primary or secondary. Primary research interest was a compulsory field at signing up. Some $43 \%$ of the users reported only one primary research interest (mean 8.94, $\mathrm{SD}=30.83$ ). Some $80 \%$ of the users did not report any secondary research interests (mean .44, $S D=3.67$ ). The minimum value for the global index was 1 and the maximum was 3,869 (mean $9.4, \mathrm{SD}=31.49$ )

There was a significant effect of position $\left(F_{(3,30424)}=9.06, p<.001, \eta_{\mathrm{p}}{ }^{2}=.001\right)$ and country development $\left(F_{(2,26534)}=64.67, p<.001, \eta_{\mathrm{p}}{ }^{2}=.005\right)$ on research interest, but no effect of ranking. Post-hoc analysis indicated that the independent researchers reported more interests than users from any other position, and faculty members followed more research interests than post-docs and graduate students. The effect of country development followed the same scale with significant intervals between very-high developed countries, who reported more research interests, to high, and finally medium-low developed countries. Among the very-high developed countries, there was an effect of location on the number of research interests $\left(F_{(2,19032)}=8.8, p<.001, \eta_{\mathrm{p}}{ }^{2}=.001\right)$. Users working in Europe reported more research interests than users in North America.

\section{Openness to contact}

Openness to contact was computed by adding 1 mark for the presence of homepage, address, phone and Skype ID and subtracting 2 marks for the absence of email. This choice was due to the fact that $e$-mail was a compulsory field at sign up and it was a public field by default. Users must intentionally change their default settings for their email address to be private. Hence, the absence of this information was considered a negative indicator of openness to contact. Some $14 \%$ of the users changed their email to private. Users most often disclosed their homepage address (18\%), address (9\%), phone (6\%), and Skype ID (4\%).

There was a significant effect of position $\left(F_{(3,30424)}=572.4, p<.001, \eta_{\mathrm{p}}{ }^{2}=.05\right)$, country development $\left(F_{(2,26534)}=73.08, p<.001, \eta_{\mathrm{p}}{ }^{2}=.005\right)$, and ranking $\left(F_{(1,27570)}\right.$ $\left.=46.54, p<.001, \eta_{\mathrm{p}}{ }^{2}=.002\right)$ on openness to contact. People affiliated to ranked universities shared more contact information than people affiliated to non-ranked universities. Post-hoc tests indicated that the effect of position was due to the same scale, previously reported, with significant intervals between faculty members, who shared more contact information, post-docs, graduate students and independent researchers. The effect of country development was due to users affiliated in very high and high developed countries, who were more open to contact than users in medium-low developed countries. Considering just very high developed countries, an effect of location was found $\left(F_{(2,19032)}=58.3, p<.001, \eta_{\mathrm{p}}{ }^{2}=.006\right)$. 
Post-hoc test indicated that users affiliated to universities in very high developed countries in Europe were more open to contact than users in very high developed countries in North America and the rest of the world.

\section{Popularity}

The average number of contacts followed by a user was $9.67(\mathrm{SD}=30.15)$ and the average number of contacts following a user was $10.89(\mathrm{SD}=22.62)$. There was a significant correlation between the number of contacts followed by and following a user $(\mathrm{r}=.61 \mathrm{p}<.01)$. The average number of colleagues was $12.59(\mathrm{SD}=19.62)$. A popularity index was computed by subtracting the number of people followed by a user from the number of followers of that user. The average popularity index was $1.21(\mathrm{SD}=25.21)$.

The Anovas indicated a significant effect of position $\left(F_{(3,30424)}=96.56, p<.001\right.$, $\left.\eta_{\mathrm{p}}{ }^{2}=.01\right)$, country development $\left(F_{(5,26534)}=35.7, p<.001, \eta_{\mathrm{p}}{ }^{2}=.003\right)$, and ranking $\left(F_{(1,27570)}=61.88, p<.001, \eta_{\mathrm{p}}{ }^{2}=.002\right)$. The effect of position reflected the scale dividing faculty members by post-docs, graduate students and independent researchers. As regards location, users affiliated with very-high developed countries were more popular than users affiliated with high and medium-low developed countries. No significant effect between researchers in the US and Europe was found. Users affiliated to ranked universities were more popular than users affiliated to universities with no ranking.

\section{Interaction among users}

A very small number of people followed, asked, and answered questions. The maximum number of questions asked by a user was 13; the maximum number of questions answered by a user was 11 ; and the maximum number of questions followed by a user was $41^{4}$. There was a positive correlation but weak between the number of asked and answered questions, $\mathrm{r}=0.23 ; \mathrm{p}<.001$.

The Anovas returned a significant effect of ranking on number of asked $\left(F_{(1,}\right.$ 27570) $\left.=12.29, p<.001, \eta_{\mathrm{p}}{ }^{2}=.000\right)$, answered $\left(F_{(1,27570)}=16.93, p<.001, \eta_{\mathrm{p}}{ }^{2}=\right.$ $.001)$, and followed questions $\left(F_{(1,27570)}=20.81, p<.001, \eta_{\mathrm{p}}{ }^{2}=.001\right)$. Users affiliated with ranked universities interacted more than those in non-ranked universities. No significant effect of position was found on number of asked questions or of followed questions. A significant effect of position was found on number of answered questions $\left(F_{(3,30424)}=6, p<.001, \eta_{\mathrm{p}}{ }^{2}=.001\right)$. Faculty members answered

\footnotetext{
${ }^{4}$ In Academia.edu, users who asked or answered a question, also follow that question by default.
} 
more questions than graduate students and independent researchers, but not of post-docs.

There was a significant effect of country development on the number of asked $\left(F_{(2,26534)}=19.8, p<.001, \eta_{\mathrm{p}}{ }^{2}=.001\right)$, answered $\left(F_{(2,26534)}=27.82, p<.001, \eta_{\mathrm{p}}{ }^{2}=\right.$ $.002)$, and followed questions $\left(F_{(2,26534)}=30.07, p<.001, \eta_{\mathrm{p}}{ }^{2}=.002\right)$. Post-hoc test indicated that all effects were due to users affiliated in very-high developed countries who asked, answered, and followed more questions than users affiliated in high and medium-low development countries. No significant effect between Europe and North America emerged.

\section{Level of engagement}

To measure the level of engagement with the network we computed the Day's interval between the first and the last update. This value was equal to zero for $45 \%$ of the sample, showing that almost half of the sample did never modified their initial profile. Among the users who did some changes, the average day's interval was 3,23.3 ( $\mathrm{SD}=245.17)$ with a maximum of 1,072 days between the first and the last update. The average number of updates per user was $5.6(\mathrm{SD}=6.2)$ with a minimum of 0 and a maximum of 25 updates. There was a positive correlation between the number of updates and interval between the date of the first and the last update, $\mathrm{r}=0.49 ; \mathrm{p}<.01$.

This level of engagement varied as a function of position $\left(F_{(3,30424)}=264.61, p<\right.$ $\left..001, \eta_{\mathrm{p}}{ }^{2}=.03\right)$, country development $\left(F_{(2,26534)}=30.07, p<.001, \eta_{\mathrm{p}}{ }^{2}=.002\right)$, and ranking $\left(F_{(1,27570)}=258.56, p<.001, \eta_{\mathrm{p}}{ }^{2}=.01\right)$. Post-hoc tests indicated that postdocs and faculty members had higher engagement level than graduate students, who are significantly more engaged than independent researchers. Users affiliated with very-high developed countries had higher level of engagement than users in high and medium-low developed countries (all difference significant). Considering just very-high developed countries, an effect of location was found $\left(F_{(2,19032)}=\right.$ $\left.34.44, p<.001, \eta_{\mathrm{p}}{ }^{2}=.004\right)$. Users affiliated with universities in Europe were more engaged than users in North America and the rest of the world. The effect of university ranking was due to users affiliated to ranked universities, who had higher level of engagement than users affiliated to non-ranked universities.

\section{Discussion}

A summary of results is reported in Table 3. A number of significant results were found and despite being small effect size they all reflect the same trend, namely the hierarchical structure of real-life academia, where position, reputation, and availability of funding (a factor strongly associated to country development) play 
a major role on people's visibility, contribution to the community, and integration within the network. For instance, faculty members (the highest hierarchical position in the University system) shared more personal and contact information than users in any other position. They also contributed with more content, and interacted with users by answering questions. In some cases, the difference between faculty members and post-docs was not significant (i.e., number of answered questions, level of engagement), but the gap was always significant between them and people at the beginning of their career (e.g., PhD students) and people who were likely to have less institutional support (e.g., independent researchers).

\begin{tabular}{|c|c|c|c|}
\hline & Position & Ranking & Country Development \\
\hline Personal information & $\begin{array}{l}\text { Faculty member (FM)- } \\
\text { Post-doc (PD)- } \\
\text { Graduate student (GS)- } \\
\text { Independent researcher (IR)* }\end{array}$ & $\begin{array}{l}\text { Ranked } \\
(\mathrm{R}) \text { - } \\
\text { Non ranked } \\
(\mathrm{NR}) *\end{array}$ & $\begin{array}{l}\text { Very high-developed } \\
\text { (VH)- } \\
\text { High develop. (H)/ Medi- } \\
\text { um-Low develop. (ML)* }\end{array}$ \\
\hline Content contribution & FM-PD-GS/IR * & R-NR * & VH-H-ML * \\
\hline Research interests & IR-FM-PD/GS * & No effect & VH-H-ML * \\
\hline Openness to contact & FM-PD-GS-IR * & $\mathrm{R}-\mathrm{NR} *$ & VH/H-ML * \\
\hline Popularity & FM- PD- GS- IR * & $\mathrm{R}-\mathrm{NR} *$ & VH-H/ML * \\
\hline Asked questions & No effect & $\mathrm{R}-\mathrm{NR} *$ & VH-H/ML * \\
\hline Answered questions & FM/PD-GS/IR * & No effect & VH-H/ML * \\
\hline Followed questions & No effect & $\mathrm{R}-\mathrm{NR} *$ & VH-H/ML * \\
\hline Level of engagement & PD/FM-GS-IR * & $\mathrm{R}-\mathrm{NR} *$ & VH-H/ML * \\
\hline
\end{tabular}

Table 3. Summary of results by variable and factor. Small $(*)$, medium $(* *)$, $\operatorname{large}(* * *)$ effect

Independent researchers were the least popular users, since they were the group with the largest difference between the number of followed contacts and the number of contacts following them, possibly reflecting the lack of an institutional network provided by the academic environment. Initially, we expected that independent researchers could try to compensate the lack of workplace connections by exposing themselves more than other users on the virtual network to bridge and bond social capital [17]. However, they were found to be the least open to share personal and contact information. Independent researchers just scored high in number of research interests, being the group following the most of them, probably reflecting the higher variability of professional life as compared to academics one. However, these results might suggest an interesting self-presentation strategy: research interests may not be used only as a source of information but also as a way to disclose information about themselves. The definition of research interest may be used as a means to establish common ground, a fundamental element in fostering cooperation between participants, especially when little is known about them [10].

Following real life trends, users affiliated to ranked universities scored higher in all variables than users in non-ranked universities. Similarly, users in very high 
developed countries scored higher than users in high and low-medium developed countries, reflecting a difference in economical possibilities between them. An interesting difference was found between North American and European researchers. Users affiliated in Europe were more open to share personal and contact information. Europeans contributed more content than users from any North American affiliation, possibly indicating a need for self-affirmation in an academic world where the old continent is still substantially less represented than North America. European users followed more research interests, which might reflect the higher specialization of North American professionals.

\section{Conclusion}

This study presents a number of limitations. First, the results are only based on a quantitative approach, which is currently work in progress. Second, the available data on users' social network did not contained information on individual connections. This information would have contributed to the analysis of social dynamics related issues, such as the effect of using of a non-hierarchical online social network to target a stratified professional community. Finally, cultural and language factors linked to the use of a professional social network in English by native and non-native users, were not researched.

Organisational structures and practices are dynamic elements of any technological ecosystem. They play an influential role in the definition of requirements, and are expected to be modified by technology appropriation [14]. Although the Internet has been presented as a democratic virtual space where physical boundaries are blurred, this chapter provides evidence that professional real-life characteristics such as position, university ranking and country level of development affect the way users build and interact in their professional virtual life. In spite of the potential of public social networks for researchers to provide equal opportunities and the interest triggered within the research community, no full advantage of these technologies has been taken yet.

Our studies suggest that these systems are mainly used to host online resumes, as a virtual business card, very similar to traditional personal web pages. The problem of motivating users to participate in and contribute to is an old major theoretical and practical challenge in the design of any type of groupware [11], and social academia does not seem to escape from it.

This exploratory study provides the foundations for understanding the dynamics in social technologies for academics and researchers. Future work will focus in better understanding users' behaviour and attitude towards public social technologies in order to develop new strategies to motivate users to contribute to the community and support equal participation. 


\section{References}

[1]Ardichvili A, Page V, Wentling T (2003) Motivations and Barriers to participation in virtual Knowledge-sharing communities of practice. Journal of knowledge management, 7(1), 64-77

[2]Boyd DM, Ellison NB (2008) Social Network Sites: Definition, History, and Scholarship. Journal of Computer- Mediated Communication, 13 (1), article 11

[3]Brzozowski MJ, Sandholm T, Hogg T (2009) Effects of Feedback and Peer Pressure on Contributions to Enterprise Social Media. In Proceedings of the ACM 2009 international conference on Supporting group work (GROUP '09). ACM, New York, NY, USA, 61-70

[4]DiMicco JM, Millen DR (2008) People Sensemaking with Social Networking Sites. CHI 2008

[5]Farrell S, Lau T, Nusser S, Wilcox E, and Muller M. 2007. Socially augmenting employee profiles with people-tagging. In Proceedings of the 20th annual ACM symposium on User interface software and technology (UIST '07). ACM, New York, NY, USA, 91-100.

[6]Gangadharbatla H (2008) Facebook Me: Collective Self-Esteem, Need to Belong, and Internet Self-Efficacy as Predictors of iGeneration's Attitudes toward Social Networking Sites. Journal of Interactive Advertising, 28(2)

[7]Huysman M, Wulf V (2006) IT to support knowledge sharing in communities, towards a social capital analysis. Journal of Information Technology, 21(1), 40-51

[8]Joinson AN (2008) 'Looking at', 'Looking up' or ' Keeping up with' People? Motives and Uses of Facebook. CHI 2008.

[9]Kumar R, Novak J, Tomkins A (2006) Structure and Evolution of Online Social Networks. Proceedings of the 12th ACM SIGKDD, 106(2), 611-617

[10]Lampe C, Ellison N, Steinfield C (2006) A Familiar Face(book): Profile Elements as Signals in an Online Social Network. CHI 2007. doi:10.1145/1240624.1240695

[11]Lampe C, Wash R, Velasquez A, Ozkaya E (2010) Motivations to Participate in Online Communities. CHI 2010.

[12]Papacharissi, Z (2009). The Virtual Geographies of Social Networks: A Comparative Analysis of Facebook, LinkedIn and ASmallWorld. New Media \& Society, 11 (1-2), 199-220.

[13]Ploderer B, Howard S, Thomas P (2008) Being Online, Living Offline: The Influence of Social Ties over the Appropriation of Social Network Sites. CSCW 2008.

[14]Rhode M, (2004) Building Social Capital in an Iranian NGO Community System. In: Huysman M, Wulf V (eds) Social Capital and Information Technology. MIT Press, Cambridge

[15]Richter A, Riemer K (2009) Corporate Social Networking Sites - Modes of Use and Appropriation through Co-Evolution. 20th Australasian Conference on Information Systems.

[16]Skeels MM, Grudin J (2009) When Social Networks Cross Boundaries: A Case Study of Workplace Use of Facebook and LinkedIn. In Proceedings of the ACM 2009 international conference on Supporting group work (GROUP '09). ACM, New York, NY, USA, 95-104.

[17]Steinfield C, DiMicco JM, Ellison NB, Lampe C (2009) Bowling Online: Social Networking and Social Capital within the Organization. Proceedings of the Fourth Communities and Technologies Conference

[18]Toma CL (2010) Affirming the Self through Online Profiles: Beneficial Effects of Social Networking Sites. CHI 2010. doi:10.1145/1753326.1753588

[19]Väänänen-Vainio-Mattila K, Wäljas M, Ojala J, Segerståhl K (2010) Identifying Drivers and Hindrances of Social User Experience in Web Services. CHI 2010.

[20]Wu A, DiMicco JM, Millen DR (2010) Detecting Professional versus Personal Closeness Using an Enterprise Social Network Site. CHI 2010. doi:10.1145/1753326.1753622 